\title{
Older adults with obesity have higher risks of some micronutrient inadequacies and lower overall dietary quality compared to peers with a healthy weight, National Health and Nutrition Examination Surveys (NHANES), 2011-2014
}

\author{
Shinyoung Jun', Alexandra E Cowan ${ }^{1}$, Anindya Bhadra' ${ }^{2}$ Kevin W Dodd ${ }^{3}$, \\ Johanna T Dwyer ${ }^{4}$, Heather A Eicher-Miller ${ }^{1}$, Jaime J Gahche ${ }^{4}$, Patricia M Guenther ${ }^{5}$, \\ Nancy Potischman ${ }^{4}$, Janet A Tooze ${ }^{6}$ and Regan L Bailey ${ }^{1, *}$ (6) \\ 'Department of Nutrition Science, Purdue University, West Lafayette, IN 47907, USA: ${ }^{2}$ Department of Statistics, \\ Purdue University, West Lafayette, IN, USA: ${ }^{3}$ National Cancer Institute, National Institutes of Health, Rockville, MD, \\ USA: ${ }^{4}$ Office of Dietary Supplements, National Institutes of Health, Bethesda, MD, USA: ${ }^{5}$ Department of Nutrition and \\ Integrative Physiology, University of Utah, Salt Lake City, UT, USA: ${ }^{6}$ School of Medicine, Wake Forest University, \\ Winston-Salem, NC, USA
}

Submitted 2 October 2019: Final revision received 2 January 2020: Accepted 15 January 2020: First published online 29 May 2020

\begin{abstract}
Objective: To evaluate total usual intakes and biomarkers of micronutrients, overall dietary quality and related health characteristics of US older adults who were overweight or obese compared with a healthy weight.

Design: Cross-sectional study.

Setting: Two 24-h dietary recalls, nutritional biomarkers and objective and subjective health characteristic data were analysed from the National Health and Nutrition Examination Survey 2011-2014. We used the National Cancer Institute method to estimate distributions of total usual intakes from foods and dietary supplements for eleven micronutrients of potential concern and the Healthy Eating Index (HEI)-2015 score.

Participants: Older adults aged $\geq 60$ years ( $n$ 2969) were categorised by sex and body weight status, using standard BMI categories. Underweight individuals ( $n$ 47) were excluded due to small sample size.

Results: A greater percentage of obese older adults compared with their healthyweight counterparts was at risk of inadequate $\mathrm{Mg}$ (both sexes), $\mathrm{Ca}$, vitamin $\mathrm{B}_{6}$ and vitamin $\mathrm{D}$ (women only) intakes. The proportion of those with serum 25-hydroxyvitamin $\mathrm{D}<40 \mathrm{nmol} / 1$ was higher in obese (12\%) than in healthyweight older women (6\%). Mean overall HEI-2015 scores were 8.6 (men) and $7 \cdot 1$ (women) points lower in obese than in healthy-weight older adults. In addition, compared with healthy-weight counterparts, obese older adults were more likely to self-report fair/poor health, use $\geq 5$ medications and have limitations in activities of daily living and cardio-metabolic risk factors; and obese older women were more likely to be food-insecure and have depression.

Conclusions: Our findings suggest that obesity may coexist with micronutrient inadequacy in older adults, especially among women.
\end{abstract}

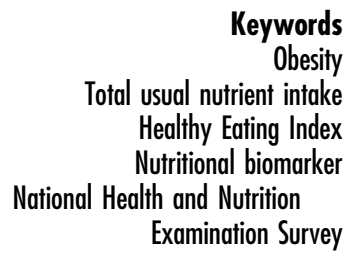

eywords

intake Healthy Eating Index Nutritional biomarker Examination Survey
The older adult population is growing rapidly. Those $\geq 65$ years currently represent approximately $15 \%$ of the US population and are projected to account for $21 \%$ in 2030, especially as 'Baby Boomers' (those born from 1946 to 1964 ) enter this life stage ${ }^{(1,2)}$. Among older adults $\geq 60$ years, the prevalence of obesity has also increased from
$31 \%$ in $2003-2004$ to $35 \%$ in $2011-2012^{(3)}$ and reached $41 \%$ in $2015-2016^{(4)}$. Obesity, especially among older adults, is of concern because it is associated with an increased risk of diet-related chronic diseases and disability ${ }^{(5,6)}$.

Adults who are obese or overweight are often assumed to consume more energy and nutrients than they require, 
but they often have inadequate intakes as well as suboptimal levels of biomarkers for some micronutrients ${ }^{(7-10)}$. Older adults who undergo age-associated changes in appetite, absorption and metabolism may have different patterns of nutrient inadequacy compared with younger counterparts $^{(11)}$. Several regional studies in the US have found that a substantial proportion of older adults, predominantly overweight or obese, are at nutrition risk ${ }^{(12,13)}$, raising awareness of the importance of identifying nutrition risks in overweight or obese older adults. However, to our knowledge, few studies have examined micronutrient status of US older adults who are obese or overweight using nationally representative data ${ }^{(14)}$. As suboptimal micronutrient status can pose threats to older adults' health and quality of life ${ }^{(11,15-17)}$, identifying unique nutritional risk in obese or overweight older adults is important to inform nutritional intervention strategies.

Therefore, in this study, using data from communityliving older adults in the National Health and Nutrition Examination Survey (NHANES) 2011-2014, we assessed micronutrient status (including total usual micronutrient intakes from diet and supplements and selected nutritional biomarkers), overall dietary quality and related sociodemographic and health characteristics among those who were overweight or obese and compared our findings with those with a healthy weight.

\section{Methods}

\section{Survey description}

NHANES is a nationally representative, continuous, cross-sectional survey of non-institutionalised civilian US residents that uses a complex, stratified, multistage probability sampling design. Detailed descriptions of the survey design and operations are available elsewhere ${ }^{(18,19)}$. Briefly, data collection occurred in three phases. Phase I included a household interview where socio-demographic and health-related information was collected using a Computer-Assisted Personal Interview system. Phase II occurred approximately 3 weeks following the household interview and included a health examination in the Mobile Examination Center, consisting of anthropometric measurements, physical examination, laboratory tests and an in-person 24-h dietary recall. Lastly, Phase III included a second 24-h dietary recall via telephone approximately 3-10 d after the health examination.

Our analytic sample combined data from the 2011-2012 and 2013-2014 NHANES cycles, the most recent survey years available for dietary supplement use data. During these cycles, Hispanics, non-Hispanic blacks and non-Hispanic Asians were over-sampled. Of the 3068 participants $\geq 60$ years who provided at least one reliable 24-h dietary recall, we excluded participants who were missing body weight or height information ( $n$ 52) and those who had BMI $\left(\mathrm{kg} / \mathrm{m}^{2}\right)<18.5$ ( $n$ 47) due to small sample size. Underweight individuals had different socio-demographic and health-related characteristics than healthy-weight individuals in our preliminary analysis; therefore, they were not combined with healthyweight individuals. Finally, our analytical sample was 2969 older adults. Sample sizes varied among characteristics of interest because certain variables (e.g. depression and low bone mass) were only collected among a subset of study participants.

\section{Antbropometric, socio-demographic and bealth-related characteristics}

Weight and height were collected by trained health technicians during the health examination in the Mobile Examination Center. BMI was calculated as weight $(\mathrm{kg})$ divided by height squared $\left(\mathrm{m}^{2}\right)$. Body weight status was classified as follows: healthy weight (BMI $18.5-24.9 \mathrm{~kg} / \mathrm{m}^{2}$ ), overweight (BMI $25-29.9 \mathrm{~kg} / \mathrm{m}^{2}$ ) and obese $\left(\mathrm{BMI} \geq 30 \mathrm{~kg} / \mathrm{m}^{2}\right)^{(20)}$.

Self-reported race and Hispanic origin were categorised as non-Hispanic white, non-Hispanic black, non-Hispanic Asian, Hispanic and other races as defined in the NHANES protocol. The 'other' race group was included in the total estimates but not reported separately. Educational attainment was categorised as less than high school, high school diploma or general equivalency diploma, some college or associate degree, and college graduate or above. The family poverty-income ratio is a ratio of annual family income to the poverty guideline established by the Department of Health and Human Services ${ }^{(21)}$. We categorised family poverty-income ratio as $\leq 130,131-350$ and $>350 \%$ because family poverty-income ratio $\leq 130 \%$ is used as a cut-off to determine financial eligibility for federal assistance programmes such as the Supplemental Nutrition Assistance Program ${ }^{(22)}$. Supplemental Nutrition Assistance Program participation status was collected at the household level and categorised as participating if the household participated in Supplemental Nutrition Assistance Program in previous 12 months. Older adults were further asked whether they received community/government meals delivered to home (e.g. Meals on Wheels) and whether they ate meals at a senior/community centre, which we combined into one variable, government/community meal participation, and dichotomised as yes or no. Adult food security status was measured using the ten adult-specific items of the U.S. Food Security Survey Module ${ }^{(23)}$. Based on the number of affirmative responses, a dichotomous adult food security variable was constructed as food-secure $(<3)$ or food-insecure (3-10).

A self-reported rating of overall health, asked via a single question in the Current Health Status questionnaire, was used in this analysis; five response categories were collapsed into three categories as either excellent/very good, good or fair/poor ${ }^{(24)}$. Self-rated overall oral health condition was also measured by a single item in the Oral Health questionnaire: 'Overall, how would you rate the 
health of your teeth and gums?' Responses were classified in the same manner as self-rated overall general health condition (i.e. excellent/very good, good or fair/poor). Depression was assessed using the Patient Health Questionnaire-9, a nine-item screening instrument that asks the frequency of depression symptoms over the past 2 weeks ${ }^{(25)}$. A total score is based on the sum of the points in each item; a score of $\geq 10$ represented depression. Participants who had missing data on one or more item were not included in the analysis for depression.

Polypharmacy was defined as taking five or more prescription medications during the $30-\mathrm{d}$ period prior to the household interview ${ }^{(26)}$. Impairment in activities of daily living and instrumental activities of daily living was defined as having any form of difficulty with the following activities: getting in or out of bed; using fork, knife and cup; walking between rooms on same floor; dressing; preparing meals; managing money or doing house chores ${ }^{(27)}$. Osteoporosis and low bone mass were determined based on the femoral neck bone mineral density using the WHO criteria: osteoporosis was defined as a T-score $\leq-2.5$ and low bone mass as a T-score between -1.0 and $-2 \cdot 5^{(28)}$. Bone mineral density information was only available in 2013-2014. We identified cardio-metabolic risk factors as present based on the criteria for metabolic syndrome components: (1) elevated waist circumference $(\geq 88 \mathrm{~cm}$ for women and $\geq 102 \mathrm{~cm}$ for men), (2) elevated blood pressure (systolic $\geq 130 \mathrm{~mm} \mathrm{Hg}$ or diastolic $\geq 85 \mathrm{mmHg}$ or both) or antihypertensive drug treatment, (3) elevated TAG $(\geq 150 \mathrm{mg} / \mathrm{dl})$ or drug treatment for elevated TAG, (4) reduced HDL-cholesterol $(<40 \mathrm{mg} / \mathrm{dl}$ for men and $<50 \mathrm{mg} /$ dl for women) or drug treatment for reduced HDL-cholesterol and (5) elevated fasting glucose ( $\geq 100 \mathrm{mg} / \mathrm{dl}$ ) or drug treatment for elevated glucose ${ }^{(29)}$.

\section{Dietary assessment and estimation of usual intake distribution}

The two 24-h recalls collected by trained NHANES interviewers probed participants on their consumption of foods and beverages as well as dietary supplements using the USDA's Automated Multiple-Pass Method ${ }^{(30)}$. Daily intakes of nutrients from foods/beverages and dietary supplements were calculated using USDA's Food and Nutrition Database for Dietary Studies and NHANES Dietary Supplement Database, respectively. We examined selected micronutrients that were identified as 'under-consumed nutrients' by the Dietary Guidelines for Americans ${ }^{(31)}$ and/or that older adults may have increased risk of inadequacy for $^{(32,33)}$ : Ca (mg), Mg (mg), $\mathrm{Zn}$ (mg), vitamin A (retinol activity equivalents; $\mathrm{mcg}$ ), folate (dietary folate equivalent; $\mathrm{mcg}$ ), vitamin $\mathrm{B}_{6}(\mathrm{mg})$, vitamin $\mathrm{B}_{12}$ ( $\left.\mathrm{mcg}\right)$, vitamin $\mathrm{C}(\mathrm{mg})$, vitamin $\mathrm{D}(\mathrm{mcg})$ and vitamin $\mathrm{E}(\boldsymbol{\alpha}$-tocopherol equivalents; mg).

Detailed information on dietary supplement use in the previous 30-d was collected during the household interview. For each dietary supplement reported, participants were asked to show containers to the trained interviewers (i.e. a product inventory) and to report information on the consumption frequency, the duration and the amount taken. If a participant used any dietary supplements during the 30-d period, the participant was classified as a supplement user.

The distributions of usual micronutrient intakes from foods alone and total intakes (i.e. foods and dietary supplements) were estimated using an adaptation of the National Cancer Institute method that produces means and standard errors and percentages of those meeting or exceeding the Dietary Reference Intakes established by the National Academies of Science, Engineering, and Mathematics, including $\%<$ estimated average requirement (EAR), \% > adequate intake (AI) and \% > tolerable upper intake level (UL). Briefly, an adaptation of the National Cancer Institute method used in this analysis can incorporate information about dietary supplements ${ }^{(34)}$; the nutrient intake from dietary supplements was added to the usual nutrient intake from foods to estimate total usual nutrient intake. These macros were previously used in the NHANES analysis by Bailey et al. ${ }^{(35)}$ Covariates in the usual intake models included day of the week of recalled day (Monday-Thursday $v$. Friday-Sunday), interview sequence of the dietary recall (first $v$. second) and dietary supplement use (yes $v$. no). Because vitamins $\mathrm{A}$ and $\mathrm{E}$ intakes from dietary supplements are not available in NHANES 20112014 , only usual intakes from foods could be determined. The estimated percentage of individuals with usual intakes below the EAR was used to indicate the percentage of those at risk for nutrient inadequacy (i.e. the EAR cut-point method) ${ }^{(36)}$. For nutrients with an AI (e.g. potassium), the percentage of individuals with intakes above the AI was used to estimate the proportion of the group at low risk of inadequacy. The estimated percentage of individuals with intakes exceeding the UL indicated the percentage who are potentially at risk of adverse effects from excess nutrient intake. The UL for folate apply only to synthetic forms obtained from supplements and fortified foods (i.e. folic acid); therefore, $\%>$ UL for folic acid was presented. The $\%>$ UL for Mg could not be determined as the UL apply only to a pharmacological agent that is not reported separately in NHANES.

The Healthy Eating Index-2015 (HEI-2015) assesses the alignment of diets with the Dietary Guidelines for Americans 2015-2020 and represents overall dietary quality $^{(37)}$. The thirteen components of the HEI-2015 include total fruits (maximum of five points), whole fruits (5), total vegetables (5), greens and beans (5), whole grains (10), dairy (10), total protein foods (5), seafood and plant proteins (5), fatty acids (10), refined grains (10), $\mathrm{Na}(10)$, added sugars (10) and saturated fats (10); details can be found in Krebs-Smith et al. ${ }^{(37)}$. Mean HEI-2015 component and total scores as well as mean total energy intakes were estimated using the Markov Chain Monte Carlo approach. 
The Markov Chain Monte Carlo method is an extension of the National Cancer Institute method that enables estimation of usual intake as well as simultaneous modelling of multiple food groups and nutrients ${ }^{(38,39)}$; the macros are publicly available ${ }^{(40)}$. The covariates included in the model were day of the week and interview sequence of the dietary recall.

\section{Nutritional biomarkers}

Whole-blood and serum samples were analysed at the CDC's Laboratory for Nutritional Biomarkers; details on specimen processing and laboratory methods are described elsewhere ${ }^{(41,42)}$. Whole-blood folate was measured by microbiologic assay, and serum folate was determined by isotope-dilution high-performance liquid chromatography coupled with tandem MS; erythrocytes folate was then calculated from the whole-blood folate by adjusting for erythrocytes volume and serum folate concentration. The cut-offs for folate deficiency used in the Dietary Reference Intake report for folate are $<7 \mathrm{nmol} / 1$ for serum folate and $<305 \mathrm{nmol} / 1$ for erythrocytes folate ${ }^{(43)}$ and were also used in this analysis. Serum vitamin $\mathrm{B}_{12}$ concentrations were assessed using electrochemiluminescence immunoassay on Elecsys, and the cut-off for vitamin $\mathrm{B}_{12}$ deficiency was $<150 \mathrm{pmol} / 1$ as recommended by the Biomarkers of Nutrition for Development expert panel ${ }^{(44)}$. Methylmalonic acid (MMA) serves as a functional measure of vitamin $B_{12}$ status and reflects vitamin $\mathrm{B}_{12}$ stores. MMA increases when serum vitamin $\mathrm{B}_{12}$ concentration is low, so $>271 \mathrm{nmol} / 1$ has been used as the cut-off for vitamin $B_{12}$ deficiency ${ }^{(44)}$. Serum concentrations of MMA were analysed by liquid chromatography with tandem MS after derivatisation with butanol. As renal insufficiency independently affects serum MMA ${ }^{(45)}$, we adjusted serum MMA estimates (both mean concentration and prevalence of deficiency) for renal function. Renal function was determined by the estimated glomerular filtration rate $\left(\mathrm{ml} / \mathrm{min}\right.$ per $\left.1.73 \mathrm{~m}^{2}\right)$ calculated from the Chronic Kidney Disease Epidemiology Collaboration (CKD-EPI) equation ${ }^{(46,47)}$ and the presence of albuminuria, defined by a urinary albumincreatinine ratio $\geq 30 \mathrm{mg} / \mathrm{g}^{(48,49)}$. Renal function was categorised as (1) normal renal function: and no albuminuria; (2) CKD stages 1-2: estimated glomerular filtration rate $\geq 60$ and single assessment of albuminuria and (3) CKD stages 3-5: estimated glomerular filtration rate $<60$ and single assessment of albuminuria. Vitamin D status was represented by serum 25-hydroxyvitamin D [25(OH)D], quantified using ultra-high-performance liquid chromatography-tandem MS. Vitamin D inadequacy was determined at $<40 \mathrm{nmol} / \mathrm{l}$ as this concentration is consistent with the $\mathrm{EAR}^{(50)}$. As vitamin $\mathrm{D}$ is produced in the skin by sunlight exposure, we adjusted vitamin $\mathrm{D}$ estimates for the season based on the month when blood was drawn: winter (November-March) and summer (AprilOctober). Lastly, although the cut-offs do not differ between racial and Hispanic origin groups for any selected biomarkers, some physiological differences by race and Hispanic origin may affect biomarker levels ${ }^{(51)}$; thus, we also presented biomarker estimates adjusted for race and Hispanic origin.

\section{Statistical analysis}

Statistical analyses were performed using SAS (version 9.4; SAS Institute, Inc.) and SAS-callable SUDAAN (version 11; RTI International) software programmes to account for the complex survey design by incorporating the NHANES 4-year Day 1 dietary sample weights. Standard errors for socio-demographic and health-related characteristics and nutritional biomarkers were constructed using Taylor series linearisation, and standard errors for HEI-2015 total and component scores and usual nutrient intakes were approximated by Fay's Modified Balanced Repeated Replication technique using thirty-two sets of replicate weights. For mean concentrations and prevalence of deficiency for MMA and vitamin D, we used logistic regression to estimate predicted marginals, adjusted for renal function (MMA) and season (vitamin D). Satterthwaite-adjusted Wald $\chi^{2}$ tests were conducted to assess differences in the distribution of socio-demographic and health-related characteristics by body weight status within sex at two-sided $P$-value of $<0.05$. Differences in percentages and means regarding nutrient biomarkers, usual nutrient intakes and HEI-2015 scores by body weight status within sex were tested using pairwise $t$ tests at a two-sided Bonferroni-adjusted $P$-value of $<0 \cdot 016$ (i.e. $0 \cdot 05 / 3$ group comparisons).

\section{Results}

Characteristics of older adults by sex and body weight status are presented in Table 1 . Obese older adults were more likely to be younger (60-69 $v$. >80 years) and less likely to be non-Hispanic Asian compared with those with a healthy weight. Obese older women, in particular, were more likely to be non-Hispanic Black, have low family income (i.e. family poverty-income ratio $\leq 130 \%$ ) and participate in Supplemental Nutrition Assistance Program when compared with healthy-weight older women. Obese older men were less likely to consume meals provided from community programmes, senior centres and/or other government programmes (e.g. Meals on Wheels) compared with healthy-weight older men. The prevalence of dietary supplement use was high regardless of weight status (61-65\% in men and $72-80 \%$ in women).

Both obese older men and women were more likely to self-report fair/poor overall health, have 3-7 limitations in activities of daily living and instrumental activities of daily living and take more than five prescription medications (i.e. polypharmacy) when compared with those with a healthy weight (Table 1). Obese older adults also had a 
Table 1 Characteristics of US older adults ( $\geq 60$ years) in the US by sex and weight status, estimated from National Health and Nutrition Examination Survey $2011-2014$

\begin{tabular}{|c|c|c|c|c|c|c|c|c|c|c|c|c|c|c|}
\hline & \multicolumn{6}{|c|}{ Men ( $n$ 1462) } & \multirow[b]{3}{*}{$P^{*}$} & \multicolumn{6}{|c|}{ Women $(n 1507)$} & \multirow[b]{3}{*}{$P$} \\
\hline & \multicolumn{2}{|c|}{$\begin{array}{l}\text { Healthy weight } \\
\text { (n 378) }\end{array}$} & \multicolumn{2}{|c|}{$\begin{array}{l}\text { Over-weight } \\
\quad(n 602)\end{array}$} & \multicolumn{2}{|c|}{ Obese ( $n$ 482) } & & \multicolumn{2}{|c|}{$\begin{array}{l}\text { Healthy weight } \\
\text { (n 399) }\end{array}$} & \multicolumn{2}{|c|}{$\begin{array}{l}\text { Over-weight } \\
\quad(n 453)\end{array}$} & \multicolumn{2}{|c|}{ Obese $(n 655)$} & \\
\hline & $\%$ & SE & $\%$ & SE & $\%$ & SE & & $\%$ & SE & $\%$ & SE & $\%$ & SE & \\
\hline \multicolumn{15}{|l|}{ Age } \\
\hline $60-69$ years & $52 \cdot 9$ & 3.8 & 55.0 & 3.0 & 63.5 & $2 \cdot 6$ & \multirow[t]{3}{*}{0.004} & $49 \cdot 0$ & 2.9 & 51.7 & $2 \cdot 3$ & $58 \cdot 3$ & $2 \cdot 3$ & \multirow[t]{3}{*}{$<0.001$} \\
\hline $70-79$ years & $27 \cdot 4$ & 3.4 & $30 \cdot 3$ & $2 \cdot 8$ & $28 \cdot 2$ & $2 \cdot 5$ & & $28 \cdot 6$ & $2 \cdot 5$ & 28.7 & $2 \cdot 6$ & 30.7 & $2 \cdot 1$ & \\
\hline $80+$ years & $19 \cdot 7$ & $2 \cdot 8$ & $14 \cdot 8$ & $1 \cdot 6$ & $8 \cdot 3$ & $1 \cdot 3$ & & $22 \cdot 5$ & $2 \cdot 1$ & $19 \cdot 6$ & $1 \cdot 8$ & $11 \cdot 1$ & $1 \cdot 6$ & \\
\hline \multicolumn{15}{|l|}{ Race and Hispanic origin } \\
\hline Non-Hispanic white & $72 \cdot 7$ & 3.3 & $79 \cdot 0$ & $2 \cdot 3$ & $80 \cdot 8$ & $2 \cdot 1$ & \multirow[t]{4}{*}{$<0.001$} & $77 \cdot 8$ & 2.5 & $80 \cdot 2$ & $2 \cdot 3$ & $72 \cdot 9$ & $2 \cdot 6$ & \multirow[t]{4}{*}{$<0.001$} \\
\hline Non-Hispanic black & $10 \cdot 8$ & 1.9 & 6.7 & $1 \cdot 0$ & 8.7 & 1.6 & & $5 \cdot 8$ & 1.4 & $7 \cdot 2$ & 1.4 & $14 \cdot 1$ & 1.9 & \\
\hline Non-Hispanic Asian & 8.5 & 1.4 & 4.0 & 0.8 & $0.6 \dagger$ & 0.3 & & 9.5 & 1.4 & 3.0 & 0.5 & 1.4 & 0.4 & \\
\hline Hispanic & $6 \cdot 6$ & 1.4 & 9.0 & 1.5 & $7 \cdot 4$ & 1.4 & & 4.8 & $1 \cdot 1$ & 8.6 & 1.5 & 9.5 & 1.8 & \\
\hline \multicolumn{15}{|l|}{ Educational attainment } \\
\hline Less than high school & $18 \cdot 3$ & $2 \cdot 5$ & $16 \cdot 2$ & $2 \cdot 0$ & $19 \cdot 6$ & $3 \cdot 1$ & \multirow[t]{4}{*}{0.14} & $16 \cdot 9$ & $2 \cdot 6$ & $20 \cdot 2$ & 3.0 & $17 \cdot 6$ & $2 \cdot 2$ & 0.35 \\
\hline High school graduate/GED or equivalent & 21.4 & $4 \cdot 1$ & 21.1 & 3.0 & 17.9 & $2 \cdot 2$ & & 21.6 & $2 \cdot 8$ & $24 \cdot 0$ & $2 \cdot 8$ & 25.5 & $2 \cdot 1$ & \\
\hline Some college or associated degree & $19 \cdot 9$ & $2 \cdot 8$ & $25 \cdot 3$ & $2 \cdot 8$ & $33 \cdot 3$ & 3.5 & & 31.7 & $2 \cdot 6$ & 33.7 & $3 \cdot 1$ & 35.9 & $2 \cdot 9$ & \\
\hline College graduate or above & 40.4 & $5 \cdot 2$ & 37.5 & 4.4 & 29.2 & $2 \cdot 8$ & & 29.9 & 3.7 & $22 \cdot 1$ & 2.5 & 21.0 & 3.0 & \\
\hline Family income & & & & & & & & & & & & & & \\
\hline $\mathrm{PIR} \leq 130 \%$ & $16 \cdot 9$ & $2 \cdot 3$ & $15 \cdot 1$ & $2 \cdot 3$ & 15.4 & 1.8 & 0.89 & $18 \cdot 4$ & $2 \cdot 7$ & $21 \cdot 3$ & $2 \cdot 4$ & $25 \cdot 6$ & $2 \cdot 6$ & 0.045 \\
\hline PIR $131-350 \%$ & $35 \cdot 6$ & 3.7 & 34.4 & $2 \cdot 6$ & $37 \cdot 0$ & $2 \cdot 1$ & & $36 \cdot 7$ & $2 \cdot 6$ & $42 \cdot 4$ & 3.6 & 41.9 & $3 \cdot 2$ & \\
\hline $\mathrm{PIR}>350 \%$ & 47.5 & $4 \cdot 8$ & 50.4 & 3.2 & 47.6 & $2 \cdot 6$ & & 44.9 & 3.3 & $36 \cdot 3$ & 3.4 & 32.5 & 3.6 & \\
\hline Food-insecurity & $7 \cdot 1$ & 1.7 & 5.6 & 1.0 & $7 \cdot 2$ & 1.4 & 0.53 & $5 \cdot 3$ & 1.4 & 6.5 & $1 \cdot 2$ & 11.5 & 1.5 & 0.009 \\
\hline Community/government meal & 8.4 & 1.7 & 5.9 & 1.2 & $3 \cdot 1$ & 0.7 & 0.011 & $8 \cdot 1$ & 1.6 & $10 \cdot 3$ & 2.0 & 9.5 & 1.9 & 0.58 \\
\hline SNAP participation & 9.3 & 1.7 & 6.0 & 1.0 & 8.9 & 1.3 & 0.09 & $9 \cdot 2$ & 1.6 & $9 \cdot 1$ & 1.6 & 14.9 & 1.9 & 0.006 \\
\hline Any dietary supplement use & $61 \cdot 1$ & 3.8 & 65.1 & $2 \cdot 7$ & $61 \cdot 8$ & 3.6 & 0.65 & 79.8 & 2.5 & 79.0 & 3.0 & 71.5 & $2 \cdot 6$ & 0.065 \\
\hline Self-rated overall health & & & & & & & & & & & & & & \\
\hline Excellent/very good & 43.4 & 3.9 & $46 \cdot 1$ & $2 \cdot 4$ & $26 \cdot 6$ & 2.5 & $<0.001$ & $51 \cdot 1$ & 3.7 & $46 \cdot 1$ & 2.5 & $34 \cdot 2$ & $2 \cdot 7$ & $<0.001$ \\
\hline Good & $37 \cdot 1$ & $3 \cdot 3$ & 38.4 & $2 \cdot 7$ & $45 \cdot 3$ & $2 \cdot 3$ & & 33.2 & 3.0 & $39 \cdot 2$ & $2 \cdot 4$ & 38.0 & $2 \cdot 4$ & \\
\hline Fair/poor & $19 \cdot 4$ & $2 \cdot 8$ & $15 \cdot 5$ & 1.5 & 28.1 & $2 \cdot 4$ & & $15 \cdot 7$ & $2 \cdot 8$ & 14.7 & 1.7 & $27 \cdot 8$ & $2 \cdot 0$ & \\
\hline Self-rated oral health & & & & & & & 0.14 & & & & & & & 0.32 \\
\hline Excellent/very good & 38.5 & 3.8 & 44.6 & $2 \cdot 7$ & 33.3 & $2 \cdot 8$ & & $47 \cdot 3$ & 3.6 & 41.9 & $2 \cdot 8$ & $41 \cdot 6$ & $2 \cdot 9$ & \\
\hline Good & $37 \cdot 1$ & 3.6 & 35.1 & $2 \cdot 1$ & 40.4 & $4 \cdot 2$ & & 31.1 & 2.9 & 37.4 & $2 \cdot 9$ & 32.5 & $2 \cdot 4$ & \\
\hline Fair/poor & 24.5 & 3.3 & 20.3 & $2 \cdot 1$ & $26 \cdot 3$ & 3.4 & & 21.7 & $3 \cdot 1$ & $20 \cdot 8$ & $2 \cdot 8$ & 25.9 & $2 \cdot 4$ & \\
\hline Depression $\ddagger$ & 8.6 & $2 \cdot 8$ & 11.3 & $2 \cdot 7$ & 11.0 & $2 \cdot 1$ & 0.74 & 11.0 & 2.6 & 7.5 & 1.6 & 19.9 & 2.5 & 0.001 \\
\hline Functional limitations in ADL and IADL & & & & & & & & & & & & & & \\
\hline No difficulty & $78 \cdot 4$ & 3.9 & 80.1 & $2 \cdot 1$ & $71 \cdot 8$ & $2 \cdot 6$ & 0.043 & $73 \cdot 0$ & 3.7 & $75 \cdot 7$ & $2 \cdot 7$ & $67 \cdot 3$ & $2 \cdot 3$ & 0.031 \\
\hline 1-2 difficulties & 12.5 & $2 \cdot 3$ & 13.8 & 1.9 & $16 \cdot 0$ & $2 \cdot 0$ & & $20 \cdot 6$ & $3 \cdot 2$ & $17 \cdot 3$ & 2.6 & $18 \cdot 6$ & $2 \cdot 1$ & \\
\hline 3-7 difficulties & $9 \cdot 1$ & $2 \cdot 3$ & 6.1 & 0.9 & $12 \cdot 2$ & 1.5 & & 6.4 & 1.2 & 7.0 & $1 \cdot 2$ & $14 \cdot 1$ & 1.7 & \\
\hline Polypharmacy ( $n$ 2517) & 31.6 & 3.7 & 30.0 & $2 \cdot 3$ & $50 \cdot 2$ & 2.9 & $<0.001$ & 28.9 & 3.7 & 40.4 & 3.7 & $51 \cdot 3$ & $2 \cdot 3$ & $<0.001$ \\
\hline Metabolic risk factors & & & & & & & & & & & & & & \\
\hline Elevated waist circumference & $3.0 \dagger$ & $1 \cdot 3$ & $57 \cdot 6$ & $2 \cdot 5$ & 98.9 & 0.7 & $<0.001$ & 33.6 & $3 \cdot 3$ & $92 \cdot 2$ & 1.7 & $100 \cdot 0$ & 0.0 & $<0.001$ \\
\hline Elevated blood Pressure & $66 \cdot 9$ & $4 \cdot 1$ & 74.2 & $2 \cdot 8$ & 85.7 & 1.8 & $<0.001$ & 64.9 & 3.9 & 79.4 & $2 \cdot 3$ & 81.3 & 1.7 & $<0.001$ \\
\hline Elevated TAG & $40 \cdot 2$ & $4 \cdot 1$ & 61.0 & 3.4 & $68 \cdot 8$ & 2.9 & $<0.001$ & 46.9 & $3 \cdot 1$ & $58 \cdot 6$ & 3.0 & 60.8 & 3.0 & 0.007 \\
\hline Reduced HDL & 40.9 & 3.9 & 66.0 & 3.2 & $69 \cdot 1$ & $2 \cdot 8$ & $<0.001$ & $40 \cdot 2$ & 3.3 & $62 \cdot 0$ & 3.0 & $66 \cdot 2$ & $2 \cdot 8$ & 0.002 \\
\hline Elevated fasting glucose & $52 \cdot 7$ & $5 \cdot 1$ & 65.8 & 4.6 & 78.7 & $2 \cdot 6$ & $<0.001$ & 44.4 & 4.6 & $58 \cdot 0$ & 3.7 & $69 \cdot 2$ & 2.5 & $<0.001$ \\
\hline Bone health§ & & & & & & & & & & & & & & \\
\hline Low bone mass & $45 \cdot 9$ & $5 \cdot 1$ & 32.4 & 3.7 & $25 \cdot 3$ & 4.1 & 0.010 & $61 \cdot 6$ & 4.1 & $59 \cdot 8$ & 3.4 & 54.4 & 5.6 & 0.49 \\
\hline Osteoporosis & 6.6 & $2 \cdot 6$ & 1.5 & 0.5 & $2 \cdot 9$ & 0.4 & 0.06 & $18 \cdot 6$ & $2 \cdot 1$ & 14.4 & $2 \cdot 8$ & $6 \cdot 1$ & 1.4 & $<0.001$ \\
\hline
\end{tabular}


Table 2 Mean Healthy Eating Index-2015 component and total scores of US older adults ( $\geq 60$ years) by sex and weight status, estimated from National Health and Nutrition Examination Survey 2011-2014

\begin{tabular}{|c|c|c|c|c|c|c|c|c|c|c|c|c|}
\hline \multirow{3}{*}{$\begin{array}{l}\text { HEI-2015 component } \\
\text { (maximum score) }\end{array}$} & \multicolumn{6}{|c|}{ Men $(n 1462)$} & \multicolumn{6}{|c|}{ Women (n 1507) } \\
\hline & \multicolumn{2}{|c|}{$\begin{array}{l}\text { Healthy weight } \\
\quad(n 378)\end{array}$} & \multicolumn{2}{|c|}{$\begin{array}{l}\text { Over-weight } \\
\quad(n \text { 602) }\end{array}$} & \multicolumn{2}{|c|}{$\begin{array}{l}\text { Obese } \\
(n 482)\end{array}$} & \multicolumn{2}{|c|}{$\begin{array}{l}\text { Healthy weight } \\
\text { (n 399) }\end{array}$} & \multicolumn{2}{|c|}{$\begin{array}{l}\text { Over-weight } \\
\quad(n \text { 453) }\end{array}$} & \multicolumn{2}{|c|}{$\begin{array}{l}\text { Obese } \\
(n 655)\end{array}$} \\
\hline & Mean & SE & Mean & SE & Mean & SE & Mean & SE & Mean & SE & Mean & SE \\
\hline Total fruits (5) & $3 \cdot 0$ & 0.3 & $2 \cdot 8$ & $1 \cdot 2$ & $2 \cdot 7$ & 0.2 & $3 \cdot 6$ & 0.2 & $3 \cdot 3$ & 0.2 & $3 \cdot 3$ & $0 \cdot 1$ \\
\hline Whole fruits (5) & 3.5 & 0.3 & 3.5 & 0.2 & 3.4 & 0.3 & $4 \cdot 2$ & 0.2 & $3 \cdot 9$ & $0 \cdot 2$ & $3 \cdot 9$ & 0.2 \\
\hline Total vegetables (5) & 3.8 & $0 \cdot 1$ & 3.7 & $0 \cdot 1$ & 3.7 & 0.2 & 4.0 & $0 \cdot 1$ & $4 \cdot 1$ & 0.1 & 3.9 & 0.1 \\
\hline Greens and beans (5) & $3 \cdot 7^{\mathrm{a}}$ & 0.4 & $3 \cdot 1^{a, b}$ & 0.3 & $2 \cdot 4^{\mathrm{b}}$ & 0.3 & 3.5 & 0.3 & $3 \cdot 1$ & 0.3 & $3 \cdot 1$ & 0.2 \\
\hline Whole grains (10) & $4 \cdot 7$ & 0.5 & $3 \cdot 6$ & 0.3 & 3.5 & 0.2 & $4 \cdot 7$ & 0.5 & 4.5 & 0.3 & 3.5 & 0.3 \\
\hline Dairy (10) & $5 \cdot 3$ & 0.3 & $5 \cdot 8$ & 0.3 & $5 \cdot 7$ & 0.3 & $6 \cdot 4$ & 0.3 & $6 \cdot 4$ & 0.3 & $6 \cdot 0$ & 0.3 \\
\hline Total protein foods (5) & 4.9 & 0.02 & $5 \cdot 0$ & 0.03 & $5 \cdot 0$ & 0.02 & 4.9 & 0.04 & 4.9 & 0.04 & 4.9 & $0 \cdot 1$ \\
\hline Seafood and plant proteins (5) & 4.6 & 0.2 & 4.4 & 0.2 & 4.5 & 0.2 & $4 \cdot 8$ & 0.1 & 4.5 & 0.2 & 4.4 & 0.2 \\
\hline Fatty acids (10) & $6 \cdot 0$ & 0.5 & $5 \cdot 2$ & 0.2 & 4.9 & 0.3 & $5 \cdot 6$ & 0.5 & 5.4 & 0.3 & $5 \cdot 2$ & 0.4 \\
\hline Refined grains (10) & $7 \cdot 6^{\mathrm{a}}$ & 0.4 & $6 \cdot 9^{a, b}$ & 0.3 & $6 \cdot 2^{\mathrm{b}}$ & 0.3 & $7 \cdot 7^{\mathrm{a}}$ & 0.3 & $7 \cdot 2^{\mathrm{a}}$ & 0.4 & $5 \cdot 6^{\mathrm{b}}$ & 0.3 \\
\hline $\mathrm{Na}(10)$ & $4.9^{\mathrm{a}}$ & 0.4 & $3 \cdot 7^{\mathrm{b}}$ & 0.3 & $2 \cdot 8^{\mathrm{b}}$ & 0.3 & $4 \cdot 5^{\mathrm{a}}$ & 0.3 & $3 \cdot 7^{\mathrm{a}, \mathrm{b}}$ & 0.2 & $3.4^{\mathrm{b}}$ & 0.3 \\
\hline Added sugars (10) & $7 \cdot 4$ & 0.3 & $7 \cdot 6$ & 0.3 & $7 \cdot 7$ & 0.3 & $7 \cdot 6$ & 0.3 & $7 \cdot 2$ & 0.2 & $7 \cdot 4$ & 0.2 \\
\hline Saturated fats (10) & $6 \cdot 9^{\mathrm{a}}$ & 0.5 & $6 \cdot 1^{a, b}$ & 0.3 & $5 \cdot 2^{b}$ & 0.3 & $6 \cdot 3$ & 0.4 & 5.9 & 0.3 & $6 \cdot 0$ & 0.3 \\
\hline Total score (100) & $66 \cdot 3^{a}$ & $2 \cdot 3$ & $61 \cdot 2^{a, b}$ & $1 \cdot 1$ & $57 \cdot 7^{b}$ & $1 \cdot 1$ & $67 \cdot 7^{a}$ & 1.8 & $64 \cdot 0^{\mathrm{a}, \mathrm{b}}$ & $1 \cdot 3$ & $60 \cdot 6^{\mathrm{b}}$ & $1 \cdot 0$ \\
\hline
\end{tabular}

${ }^{a, b}$ Mean values within a same row for each sex with different superscript letters were significantly different $(P<0.016)$.

consistently higher prevalence of the five cardio-metabolic risk factors that comprise metabolic risk syndrome (i.e. elevated waist circumference, elevated blood pressure, elevated TAG, reduced HDL-cholesterol and elevated fasting glucose levels). Among older women, the prevalence of depression was higher in the obese $(20 \%)$ than in those who were overweight ( $8 \%$ ) or had a healthy weight $(11 \%)$. Meanwhile, obese older men were less likely to have low bone mass, and obese older women were less likely to have osteoporosis, compared with their healthy-weight counterparts.

Obese older adults had lower mean total HEI-2015 scores than healthy-weight older adults; the differences were largely driven by refined grains and $\mathrm{Na}$ in both sexes, greens and beans and saturated fats in men, and whole grains in women (Table 2). No significant differences in mean total energy intake from food sources were observed across body weight status categories for either men or women (data not shown).

Based on usual nutrient intakes from foods alone, the prevalence of inadequate intakes ( $\%<$ EAR) was higher in the obese group for $\mathrm{Mg}$ (among both men and women), vitamins $\mathrm{C}$ and $\mathrm{E}$ (among men only), and vitamin $\mathrm{B}_{6}$ and vitamin D (among women only) compared with the healthy-weight group (Table 3 ). In addition, the percentage of those with potassium intakes above the AI was lower in the obese of both sexes than in the healthy weight. Even after accounting for nutrients from dietary supplements, a substantial proportion of older adults ( $>30 \%$ for at least one group) were at risk of inadequate intakes for $\mathrm{Ca}$, $\mathrm{Mg}$, vitamin C and vitamin D (Table 3). Obese older adults had a higher prevalence of inadequate $\mathrm{Mg}$ intakes compared with their healthy-weight counterparts. Obese older women were at higher risk of inadequate intakes for $\mathrm{Ca}$, vitamins $\mathrm{B}_{6}$ and vitamin $\mathrm{D}$ than those at a healthy weight.

Some individuals were at risk of excessive intakes (i.e. \% > UL) for $\mathrm{Ca}(3-13 \%)$, Zn, vitamin $\mathrm{D}$, folic acid, vitamin $\mathrm{B}_{6}$ and vitamin $\mathrm{C}(<7 \%)$ based on their total usual intakes (Fig. 1). Few differences in \% >UL were noted by body weight status, except that the prevalence of excessive total Ca intake was higher in older adults who had a healthy weight than in those who were obese. With respect to nutrient intakes from foods alone, almost no one was at risk of excessive intakes for any of the nutrients examined, except for $\mathrm{Ca}$ in approximately $5 \%$ of men, across body weight status categories (data not shown).

The percentages of those who had biomarker concentrations below recommendations are shown in Table 4. Notable difference by body weight status was found for the prevalence of vitamin D insufficiency among women; the proportion of those with serum $25(\mathrm{OH}) \mathrm{D}<40 \mathrm{nmol} / \mathrm{l}$ was higher in obese women (12\%) than in healthy-weight women $(6 \%)$. The difference did not reach the statistical significance after adjusting for race and Hispanic origin, but the relative order was preserved (Supplemental Table 1). The mean concentrations of serum 25(OH)D were significantly lower in the obese compared with those with either overweight or a healthy weight among both older men and women, regardless of adjustment for race and Hispanic origin. Almost no one was folate-deficient based on either serum folate or erythrocytes folate, and the percentage of those with serum vitamin $\mathrm{B}_{12}<150 \mathrm{pmol}$ was also very low ( $<5 \%$ ) across body weight status categories. The percentage of older adults with MMA $>271 \mathrm{nmol} / \mathrm{l}$, adjusted for renal function, was significantly lower in obese older men (11\%) compared with healthy-weight older men (19\%). 
Table 3 Prevalence of intakes less than the estimated average requirement (EAR) or above the adequate intake (AI) among US older adults ( $\geq 60$ years) by sex and weight status, estimated from National Health and Nutrition Examination Survey 2011-2014

\begin{tabular}{|c|c|c|c|c|c|c|c|c|c|c|c|c|}
\hline & \multicolumn{6}{|c|}{ Men $(n 1462)$} & \multicolumn{6}{|c|}{ Women (n 1507) } \\
\hline & \multicolumn{2}{|c|}{$\begin{array}{c}\text { Healthy } \\
\text { weight } \\
(n 378)\end{array}$} & \multicolumn{2}{|c|}{$\begin{array}{l}\text { Over-weight } \\
\quad(n \text { 602) }\end{array}$} & \multicolumn{2}{|c|}{$\begin{array}{l}\text { Obese } \\
(n 482)\end{array}$} & \multicolumn{2}{|c|}{$\begin{array}{c}\text { Healthy } \\
\text { weight } \\
\text { (n 399) }\end{array}$} & \multicolumn{2}{|c|}{$\begin{array}{l}\text { Over-weight } \\
\quad(n \text { 453) }\end{array}$} & \multicolumn{2}{|c|}{$\begin{array}{l}\text { Obese } \\
(n \text { 655) }\end{array}$} \\
\hline & $\%$ & SE & $\%$ & SE & $\%$ & SE & $\%$ & SE & $\%$ & SE & $\%$ & SE \\
\hline \multicolumn{13}{|l|}{ From food sources alone } \\
\hline $\mathrm{Ca}(\%<\mathrm{EAR})$ & $46 \cdot 0$ & $2 \cdot 8$ & 44.0 & $2 \cdot 8$ & $42 \cdot 0$ & 2.9 & 74.0 & $3 \cdot 6$ & $78 \cdot 0$ & $3 \cdot 6$ & $82 \cdot 0$ & 3.5 \\
\hline $\mathrm{Mg}(\%<\mathrm{EAR})$ & $51 \cdot 0^{\mathrm{a}}$ & 2.5 & $63 \cdot 7^{\mathrm{b}}$ & 2.9 & $73.9^{c}$ & $2 \cdot 7$ & $45 \cdot 7^{\mathrm{a}}$ & 4.6 & $57 \cdot 4^{\mathrm{a}, \mathrm{b}}$ & 3.5 & $64 \cdot 4^{\mathrm{b}}$ & $3 \cdot 1$ \\
\hline $\mathrm{K}(\%>\mathrm{Al})$ & $40 \cdot 0^{\mathrm{a}}$ & $3 \cdot 0$ & $28 \cdot 0^{b}$ & $3 \cdot 1$ & $22 \cdot 0^{b}$ & $3 \cdot 4$ & $40 \cdot 0^{a}$ & $4 \cdot 3$ & $35 \cdot 0^{\mathrm{a}, \mathrm{b}}$ & $3 \cdot 6$ & $27 \cdot 0^{b}$ & $2 \cdot 3$ \\
\hline $\mathrm{Zn}(\%<\mathrm{EAR})$ & 23.5 & 4.2 & $20 \cdot 3$ & 4.7 & $27 \cdot 2$ & 4.0 & $17 \cdot 4$ & 3.9 & $21 \cdot 1$ & 4.9 & $26 \cdot 6$ & 3.4 \\
\hline Vitamin $\mathrm{A}(\%<\mathrm{EAR})$ & 43.0 & $3 \cdot 1$ & $26 \cdot 0^{\star}$ & 14.4 & $51 \cdot 0$ & $5 \cdot 8$ & $27 \cdot 0$ & $6 \cdot 4$ & $37 \cdot 0$ & $3 \cdot 3$ & 39.0 & $5 \cdot 2$ \\
\hline Folate $(\%<$ EAR $)$ & $9 \cdot 0^{a, b}$ & 2.5 & $4 \cdot 0^{\star a}$ & $2 \cdot 0$ & $13 \cdot 0^{\mathrm{b}}$ & $3 \cdot 2$ & $16 \cdot 0$ & 3.6 & 23.0 & 4.4 & $22 \cdot 0$ & $2 \cdot \overline{8}$ \\
\hline Vitamin $\mathrm{B}_{6}(\%<\mathrm{EAR})$ & $12 \cdot 0$ & $2 \cdot 3$ & $5 \cdot 5^{*}$ & $2 \cdot 7$ & $10 \cdot 3$ & 3.2 & $18 \cdot 2^{\mathrm{a}}$ & $4 \cdot 2$ & $23 \cdot 0^{a, b}$ & $5 \cdot 1$ & $33 \cdot 7^{b}$ & $2 \cdot 7$ \\
\hline Vitamin $B_{12}(\%<$ EAR $)$ & $4 \cdot 4^{*}$ & $2 \cdot 1$ & $1 \cdot 1^{*}$ & 0.7 & $4 \cdot 4^{*}$ & 1.9 & 8.0 & $2 \cdot 7$ & $4 \cdot 9^{*}$ & $2 \cdot 1$ & 7.5 & $2 \cdot 7$ \\
\hline Vitamin $\mathrm{C}(\%<\mathrm{EAR})$ & $43 \cdot 7^{a}$ & $2 \cdot 3$ & $52 \cdot 9^{a, b}$ & $3 \cdot 8$ & $56 \cdot 3^{\mathrm{b}}$ & 3.6 & $37 \cdot 6$ & 3.9 & 38.0 & $3 \cdot 3$ & $47 \cdot 1$ & $3 \cdot 3$ \\
\hline Vitamin D (\% <EAR) & $88 \cdot 8$ & $2 \cdot 6$ & 94.8 & $2 \cdot 4$ & 94.0 & $2 \cdot 1$ & $93.0^{\mathrm{a}}$ & $2 \cdot 1$ & $97 \cdot 9^{a, b}$ & 0.9 & $98 \cdot 6^{b}$ & 0.8 \\
\hline Vitamin E (\% <EAR) & $67 \cdot 3^{\mathrm{a}}$ & 3.0 & $77 \cdot 2^{a, b}$ & $3 \cdot 8$ & $85 \cdot 6^{\mathrm{b}}$ & 3.4 & $86 \cdot 2$ & 3.4 & 91.4 & 3.0 & 91.8 & 2.4 \\
\hline \multicolumn{13}{|l|}{ From total intakes } \\
\hline $\mathrm{Ca}(\%<\mathrm{EAR})$ & $36 \cdot 0$ & $2 \cdot 8$ & 33.0 & $2 \cdot 3$ & 31.0 & $2 \cdot 7$ & 38.0 & $3 \cdot 2$ & $44 \cdot 0^{\mathrm{a}}$ & 3.5 & $56 \cdot 0^{b}$ & 3.7 \\
\hline $\mathrm{Mg}(\%<\mathrm{EAR})$ & $44 \cdot 0^{\mathrm{a}}$ & $3 \cdot 0$ & $53 \cdot 8^{\mathrm{b}}$ & $2 \cdot 6$ & $61.0^{\mathrm{b}}$ & $3 \cdot 0$ & $34.0^{\mathrm{a}}$ & $4 \cdot 3$ & $43 \cdot 0^{a, b}$ & $3 \cdot 2$ & $52 \cdot 6^{\mathrm{b}}$ & $3 \cdot 2$ \\
\hline $\mathrm{K}(\%>\mathrm{Al})$ & $41 \cdot 0^{\mathrm{a}}$ & $3 \cdot 0$ & $29 \cdot 0^{\mathrm{b}}$ & $3 \cdot 1$ & $24 \cdot 0^{b}$ & $3 \cdot 3$ & $42 \cdot 0^{a}$ & $4 \cdot 3$ & $36 \cdot 0^{a, b}$ & 3.5 & $28 \cdot 0^{b}$ & $2 \cdot 3$ \\
\hline $\mathrm{Zn}(\%<\mathrm{EAR})$ & $16 \cdot 4$ & $3 \cdot 2$ & 14.4 & 3.5 & 18.5 & 3.7 & 11.0 & $2 \cdot 7$ & $12 \cdot 0$ & $3 \cdot 0$ & $19 \cdot 6$ & $2 \cdot 7$ \\
\hline Folate $(\%<\mathrm{EAR})$ & $7 \cdot 0$ & $2 \cdot 1$ & $3 \cdot 0^{*}$ & $1 \cdot 7$ & 8.0 & $2 \cdot 0$ & $10 \cdot 0$ & $2 \cdot 5$ & $12 \cdot 0$ & $2 \cdot 8$ & $17 \cdot 0$ & $2 \cdot 4$ \\
\hline Vitamin $\mathrm{B}_{6}(\%<\mathrm{EAR})$ & $8 \cdot 6$ & $2 \cdot 1$ & $4 \cdot 6^{*}$ & $2 \cdot 2$ & $6 \cdot 0$ & $2 \cdot 1$ & $12 \cdot 5^{\mathrm{a}}$ & $3 \cdot 2$ & $13 \cdot 6^{\mathrm{a}}$ & $2 \cdot 7$ & $22 \cdot 8^{\mathrm{b}}$ & $2 \cdot 7$ \\
\hline Vitamin $\mathrm{B}_{12}(\%<\mathrm{EAR})$ & 3.0 & $1 \cdot 2$ & $1 \cdot 0^{*}$ & 0.5 & $3 \cdot 0^{*}$ & 1.4 & 4.0 & 1.0 & $2 \cdot 0^{\star}$ & 1.0 & $6 \cdot 0$ & $2 \cdot 0$ \\
\hline Vitamin $\mathrm{C}(\%<\mathrm{EAR})$ & $29 \cdot 0$ & 4.4 & $37 \cdot 0$ & $3 \cdot 6$ & $32 \cdot 0$ & $3 \cdot 0$ & $24 \cdot 0^{\mathrm{a}, \mathrm{b}}$ & 4.0 & $21 \cdot 0^{\mathrm{a}}$ & $3 \cdot 1$ & $31.0^{\mathrm{b}}$ & $2 \cdot 3$ \\
\hline Vitamin $\mathrm{D}(\%<\mathrm{EAR})$ & $48 \cdot 0$ & 3.9 & $53 \cdot 1$ & $2 \cdot 8$ & $51 \cdot 1$ & $3 \cdot 3$ & $33.0^{\mathrm{a}}$ & $3 \cdot 1$ & $39 \cdot 4^{\mathrm{a}, \mathrm{b}}$ & 3.4 & $49 \cdot 4^{\mathrm{b}}$ & 3.2 \\
\hline
\end{tabular}

${ }^{*}$ Relative standard error over $40 \%$; estimates may not be statistically reliable.

a,b,c Mean values within a same row for each sex with different superscript letters were significantly different $(P<0.016)$

\section{Discussion}

These nationally representative results indicate that a considerable proportion of older adults with $\mathrm{BMI} \geq 18.5 \mathrm{~kg} / \mathrm{m}^{2}$ were at risk of inadequate total intakes for $\mathrm{Ca}, \mathrm{Mg}$, vitamin $\mathrm{C}$ and vitamin D. Obese older adults had similar or even higher risks of inadequate intakes for all micronutrients examined compared with those with lower BMI, and this finding was especially evident among women. Very limited national estimates are available on total usual micronutrient intakes of US older adults by body weight status. Given that dietary supplement use is very high in this population ${ }^{(52)}$, nutrient intake assessment without including dietary supplements would be incomplete ${ }^{(53-55)}$. Across body weight status categories, nutrients from dietary supplements not only contributed to reducing the prevalence of inadequate intakes for all micronutrients examined but also led to small increases in the prevalence of excessive intakes among US older adults. Interestingly, after inclusion of dietary supplements, fewer differences by body weight status were observed in older men, but more differences were observed in older women.

Our results on usual micronutrient intakes from foods alone are consistent with those from another study among US adults ( $\geq 19$ years, men and women combined) where the obese had higher risks of inadequate intakes for vitamins $\mathrm{A}, \mathrm{C}, \mathrm{D}$, and $\mathrm{E}, \mathrm{Ca}$, and $\mathrm{Mg}$ and lower HEI-2005 scores than those with a healthy weight ${ }^{(56)}$. In this study among older adults, a greater percentage of the obese had intakes that did not meet the EAR for Mg (both sexes), folate and vitamins $\mathrm{C}$ and $\mathrm{E}$ (men), and vitamins $\mathrm{B}_{6}$ and $\mathrm{D}$ (women) and the AI for potassium (both sexes), when compared with those of healthy weight. Poorer overall dietary quality may have contributed to this; obese older adults had lower scores for total HEI-2015 and its several components, such as greens and beans (men) and whole grains (women). Lower scores for moderation components such as refined grains and saturated fats also raise concerns about meeting nutrient requirements within energy needs.

It is noteworthy that the prevalence of inadequate $\mathrm{Mg}$ intake was consistently higher in obese older adults than in healthy-weight peers, regardless of sex and inclusion of dietary supplements. Although clinical signs of overt $\mathrm{Mg}$ deficiency are rare, inadequate $\mathrm{Mg}$ intakes have been associated with chronic inflammatory response $e^{(57)}$ and several chronic diseases, including CVD, type 2 diabetes mellitus and neurological disorders ${ }^{(58)}$. Older adults have lower dietary intakes of $\mathrm{Mg}$ than younger adults ${ }^{(55)}$ despite decreased intestinal absorption and increased urinary excretion with age ${ }^{(59)}$. This finding indicates that older adults with obesity may be at particular risk of $\mathrm{Mg}$ inadequacy. 
(a) Men

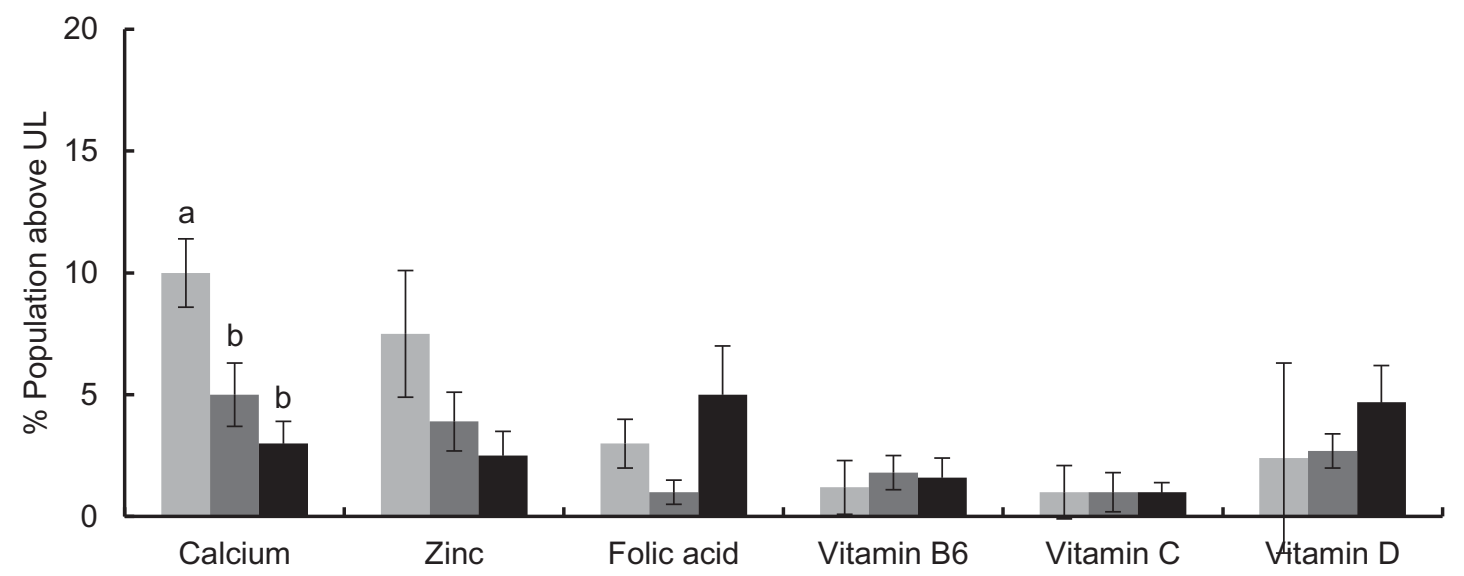

(b) Women

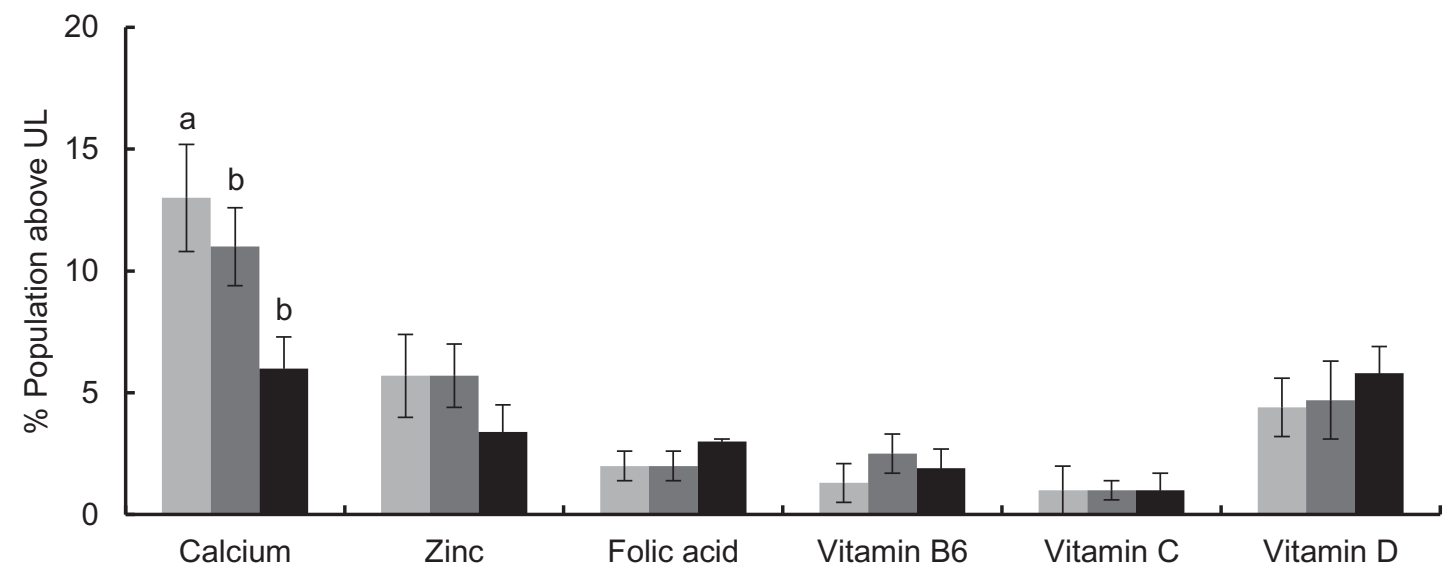

Fig. 1 Prevalence of total usual micronutrient intakes above the Tolerable Upper Intake Level (UL) among US older adults ( $\geq 60$ years) by sex and weight status, estimated from National Health and Nutrition Examination Survey (NHANES) 2011-2014: (A) men; (B) women. The UL for folate apply to folic acid, which is a synthetic form obtained from supplements, fortified foods or a combination of the two. Estimates for vitamins $A$ and $E$ could not be determined as intakes from dietary supplements are not available in NHANES 2011-2014. ${ }^{\text {a,b }}$ Mean values within a same row for each sex with different superscript letters were significantly different $(P<0.016)$. (A and $\mathrm{B}) \square$, Healthy weight; $\square$, over weight; $\boldsymbol{\square}$, obese

Self-reported dietary data are well-known to include bias coming from errors in memory and portion size estimation $^{(60)}$, and obese individuals may under-report their energy and protein intakes ${ }^{(61)}$. Under-reporting of energy intakes is also likely to be associated with under-reporting of nutrient intakes, but much less is known about nutrients without recovery biomarkers. Biomarkers that are independent of reporting bias and may reflect absorption and metabolism can provide complementary information on nutritional status.

In accordance with our results on total vitamin D intake, a greater proportion of older women had inadequate vitamin $\mathrm{D}$ status (i.e. $25(\mathrm{OH}) \mathrm{D}<40 \mathrm{nmol} / \mathrm{l}$ ) than those with a healthy weight after adjusting for season of specimen collection. A lower concentration of $25(\mathrm{OH}) \mathrm{D}$ in the obese has been consistently reported among adults of various ages ${ }^{(7,62-65)}$, although the cause is unknown. Possible mechanisms include lower dietary intake ${ }^{(66)}$, differential sun exposure, altered metabolism such as sequestration in adipose tissue and/or volumetric dilution ${ }^{(67)}$. For example, Drincic et al. (2012) found that 25(OH)D in obese individuals was mostly explained by body volume (i.e. volumetric dilutional model) ${ }^{(68)}$. In addition, physiologic differences between races and Hispanic origins (e.g. skin pigmentation) may contribute to, but not completely explain, the differences in the prevalence of inadequate vitamin D status by weight status in women ${ }^{(51)}$. Whether obese individuals have higher requirements for vitamin D or need a different $25(\mathrm{OH}) \mathrm{D}$ cut-off is yet to be determined. Moreover, the obese had lower risk of suboptimal bone mass despite low $25(\mathrm{OH}) \mathrm{D}$ concentrations in this and other cross-sectional analyses ${ }^{(65)}$. Further studies are warranted to determine the best strategies to achieve optimal vitamin D status among obese older adults.

The prevalence of folate deficiency based on serum and erythrocytes folate was very low $(<2 \%)$ among US older 


\section{N Public Health Nutrition}

Table 4 Status of US older adults ( $\geq 60$ years) for selected nutritional biomarkers by sex and weight status, estimated from National Health and Nutrition Examination Survey (NHANES) $2011-2014$

\begin{tabular}{|c|c|c|c|c|c|c|c|c|c|c|c|c|}
\hline & \multicolumn{6}{|c|}{ Men $(n 1462)$} & \multicolumn{6}{|c|}{ Women $(n 1507)$} \\
\hline & \multicolumn{2}{|c|}{$\begin{array}{l}\text { Healthy weight } \\
\text { (n 378) }\end{array}$} & \multicolumn{2}{|c|}{ Over-weight ( $n$ 602) } & \multicolumn{2}{|c|}{ Obese ( $n$ 482) } & \multicolumn{2}{|c|}{$\begin{array}{l}\text { Healthy weight } \\
\text { (n 399) }\end{array}$} & \multicolumn{2}{|c|}{ Over-weight ( $n$ 453) } & \multicolumn{2}{|c|}{ Obese $(n$ 655) } \\
\hline & $\%$ or Mean & SE & $\%$ or Mean & SE & $\%$ or Mean & SE & $\%$ or Mean & SE & $\%$ or Mean & SE & $\%$ or Mean & SE \\
\hline \multicolumn{13}{|l|}{ Serum $25(\mathrm{OH}) \mathrm{D}^{*}$} \\
\hline$\%<40 \mathrm{nmol} / \mathrm{l}$ & 6.9 & 1.4 & $5 \cdot 4$ & $1 \cdot 3$ & $8 \cdot 7$ & $2 \cdot 0$ & $5 \cdot 6^{a}$ & 1.6 & $7 \cdot 0^{\mathrm{a}, \mathrm{b}}$ & $2 \cdot 0$ & $11 \cdot 7^{\mathrm{b}}$ & 2.0 \\
\hline Concentration (nmol/l) & $81 \cdot 3^{\mathrm{a}}$ & 1.6 & $78 \cdot 3^{\mathrm{a}}$ & 1.4 & $72 \cdot 6^{\mathrm{b}}$ & $1 \cdot 7$ & $93 \cdot 4^{\mathrm{a}}$ & $3 \cdot 0$ & $87 \cdot 4^{\mathrm{a}}$ & $2 \cdot 6$ & $78 \cdot 4^{\mathrm{b}}$ & 1.6 \\
\hline \multicolumn{13}{|l|}{ Serum folate } \\
\hline$\%<7 \mathrm{nmol} / \mathrm{l}$ & $0.1 \dagger$ & $0 \cdot 1$ & 0.0 & 0.0 & 0.0 & 0.0 & 0.0 & 0.0 & $0.2 \dagger$ & 0.2 & $0.1 \dagger$ & 0.1 \\
\hline Concentration (nmol//) & 56.5 & $2 \cdot 9$ & $51 \cdot 3$ & $1 \cdot 3$ & $52 \cdot 8$ & 2.5 & $65 \cdot 1^{\mathrm{a}}$ & 1.9 & $67.9^{\mathrm{a}}$ & $2 \cdot 7$ & $56 \cdot 0^{\mathrm{b}}$ & $1 \cdot 7$ \\
\hline \multicolumn{13}{|l|}{ Erythrocytes folate } \\
\hline$\%<305 \mathrm{nmol} / \mathrm{l}$ & $1.7 \dagger$ & 1.4 & $0.1 \dagger$ & 0.1 & 0.0 & 0.0 & $0.7 \dagger$ & 0.5 & $0.1 \dagger$ & 0.1 & $0.1 \dagger$ & 0.1 \\
\hline Concentration (nmol//) & $1323^{a}$ & 53 & $1335^{\mathrm{a}, \mathrm{b}}$ & 33 & $1464^{b}$ & 42 & $1432^{\mathrm{a}}$ & 34 & $1586^{b}$ & 44 & $1496^{\mathrm{a}, \mathrm{b}}$ & 44 \\
\hline \multicolumn{13}{|l|}{ Serum vitamin $B_{12}$} \\
\hline$\%<150 \mathrm{pmol} / \mathrm{l}$ & $2 \cdot 7^{\mathrm{a}} \dagger$ & $1 \cdot 1$ & $4 \cdot 3^{\mathrm{a}}$ & $1 \cdot 3$ & $3.4^{\mathrm{a}} \dagger$ & 1.7 & $1.0^{\mathrm{a}} \mathrm{t}$ & 0.7 & $2 \cdot 5^{a, b}$ & 0.9 & $3 \cdot 2^{b}$ & 0.8 \\
\hline Concentration (pmol/l) & 513.7 & $35 \cdot 7$ & $435 \cdot 8$ & $13 \cdot 2$ & 422.6 & $17 \cdot 1$ & 630.4 & $37 \cdot 5$ & $562 \cdot 0$ & $25 \cdot 7$ & $560 \cdot 6$ & $53 \cdot 3$ \\
\hline \multicolumn{13}{|l|}{ Serum MMA } \\
\hline$\%>271 \mathrm{nmol} / \mathrm{l}$ & $19 \cdot 3^{\mathrm{a}}$ & $3 \cdot 0$ & $12 \cdot 9^{a, b}$ & 2.5 & $11 \cdot 1^{b}$ & $2 \cdot 1$ & $14 \cdot 8$ & $2 \cdot 1$ & $12 \cdot 0$ & 1.5 & $15 \cdot 8$ & $2 \cdot 4$ \\
\hline Concentration (nmol/l) & $210 \cdot 2$ & $13 \cdot 1$ & $201 \cdot 0$ & $7 \cdot 4$ & $195 \cdot 3$ & 11.4 & $198 \cdot 0$ & $7 \cdot 4$ & $185 \cdot 6$ & $6 \cdot 3$ & $204 \cdot 0$ & $10 \cdot 6$ \\
\hline
\end{tabular}

25(OH)D, 25-hydroxyvitamin D; MMA, methylmalonic acid; CKD, chronic kidney disease.

*Adjusted for season (winter or summer).

†Relative SE over $40 \%$; estimates may not be statistically reliable.

CKD stages 1-2, and CKD stages 3-5).

a,b Mean values within a same row for each sex with different superscript letters were significantly different $(P<0.016)$. 
adults, but the prevalence of inadequate total folate intake was 3-8\% in men and $10-17 \%$ in women; this discrepancy has been previously documented ${ }^{(69)}$. Mean serum folate concentration was lower in obese women $\geq 60$ years than in those with a healthy weight, as were reported by studies on women of various ages ${ }^{(7,70-72)}$. Our finding also adds evidence on a positive relation between BMI and erythrocytes folate in older adults, which was previously observed in younger adults 19-50 years but not in those $>50$ years ${ }^{(70)}$. Our estimates indicate that a considerable proportion of older adults (11-19\%) had vitamin $B_{12}$ deficiency based on serum MMA concentrations. However, it should be noted that serum MMA concentrations increase with age even in those with replete vitamin $\mathrm{B}_{12}$ status and normal renal function and age-specific MMA reference ranges were suggested recently ${ }^{(73)}$. In this analysis, vitamin $\mathrm{B}_{12}$ deficiency based on serum MMA was more common in obese older men than in healthy-weight counterparts after adjusting for renal function, whereas some studies have suggested an inverse association between serum $\mathrm{B}_{12}$ concentrations and $\mathrm{BMI}^{(74,75)}$. Further investigation is needed to determine the relationship of serum $\mathrm{B}_{12}$ and MMA concentrations with BMI.

Many factors can influence nutritional health. Shlisky et al. ${ }^{(76)}$ identified frailty; age-related disease; and sociologic, biological, and cognitive issues as negative factors and social support and economic status; healthcare; and diet resilience (i.e. ability to adapt strategies to maintain a high-quality diet despite challenges) as positive factors that influence nutritional health for healthy ageing. Our results suggest that older adults who are overweight or obese have poorer health subjectively as well as objectively, represented by cardio-metabolic risk factors and polypharmacy, compared with those with a healthy weight. Whether micronutrient inadequacy increased the risk of obesity-related chronic diseases needs further investigation $^{(77)}$. Among older women, body weight status was associated with depression, lower family income and food insecurity; taken together, these data suggest that obese US older women, in particular, may be at nutritional risk.

The findings of this study should be interpreted cautiously with several limitations in mind. As mentioned above, overweight/obesity status has been associated with under-reporting for $24-\mathrm{h}$ recalls ${ }^{(60,61)}$. To reduce the measurement error, NHANES employs the state-of-the-art instrument for dietary intake assessment, the Automated Multiple-Pass Method ${ }^{(78)}$, and staffs review the reliability of each recall. In addition, data on longer-term dietary supplement use (the prior $30 \mathrm{~d}$ ) collected from dietary supplement questionnaire in tandem with an in-home inventory were combined with data from 24-h recalls. We also presented biomarker data to complement selfreport dietary data, but biomarkers examined in this study are concentration biomarkers that can be affected by personal characteristics, and cut-offs can be arbitrary and vary even for the same biomarker ${ }^{(79)}$. Lastly, BMI may not always reflect body composition, which is particularly challenging in older adults due to age-related changes in lean body mass, body fat and height ${ }^{(80)}$. The strengths of this study include the nationally representative sample of community-dwelling older adults and estimation of distributions of usual intakes of nutrients and usual HEI2015 scores.

In conclusion, comprehensive information on anthropometric, clinical and dietary intake suggests that obesity in older adults coexists with micronutrient inadequacies. Older adults with obesity had similar or higher risk of inadequate intakes for some micronutrients (e.g. $\mathrm{Mg}$ in both sexes, and $\mathrm{Ca}$, vitamin $\mathrm{B}_{6}$, and vitamin $\mathrm{D}$ in women only), higher prevalence of vitamin $\mathrm{D}$ inadequacy based on serum $25(\mathrm{OH}) \mathrm{D}$ concentrations and lower overall dietary quality, compared with those with a healthy weight. Moreover, obese older adults were more likely to self-report poor health, take more medications and have multiple functional limitations and cardio-metabolic risk factors, all of which can increase nutritional risk. Obese older adults should be particularly careful to meet micronutrient recommendations by choosing a nutrient-dense diet and/or judicious use of dietary supplements when food sources of nutrients alone are not enough.

\section{Acknowledgements}

Financial support: This work was supported by the NIH/NCI U01CA215834. NIH had no role in the design, analysis or writing of this article. Conflict of interest: S.J., A.E.C., A.B., K.W.D., H.A.E-M., J.G., P.M.G., N.P. and J.A.T. have no conflicts of interest. R.L.B. serves as a scientific consultant to the National Institute of Health, Office of Dietary Supplements; has received travel support from the Council of Responsible Nutrition, American Society of Nutrition, and New York Academy of Sciences to present her research on nutrient intakes and supplementation; has served as a consultant to Columbia University on a paper on nutrient intakes and RTI International on 2016 Feeding Infants and Toddlers Study data (funded by Nestle R\&D). J.T.D. reports personal fees from McCormick Spice, personal fees from The Mushroom Council, personal fees from Bay State Milling, personal fees from ConAgra Foods, personal fees from Nestlé, outside the submitted work. Authorship: R.L.B. and S.J. designed research; S.J. analysed data and drafted the manuscript; S.J. and R.L.B. had primary responsibility for final content; all authors participated in the interpretation of the data and critical revision of the manuscript. All authors have read and approved the final manuscript. Ethics of buman subject participation: This study was conducted according to the guidelines laid down in the Declaration of Helsinki. NHANES procedures were approved by the Research Ethics Review Board at the National Center for Health 
Statistics/Centers for Disease Control and Prevention, and written informed consent was obtained from all participants or proxies.

\section{Supplementary material}

For supplementary material accompanying this paper visit https://doi.org/10.1017/S1368980020000257

\section{References}

1. Federal Interagency Forum on Aging-Related Statistics (2016) Older Americans 2016: Key Indicators of Well-being. Washington, DC: U.S. Government Printing Office.

2. Vincent GK \& Velkoff VA (2010) The Next Four Decades: The Older Population in the United States: 2010 to 2050. US Department of Commerce, Economics and Statistics Administration. Washington, DC: US Census Bureau.

3. Ogden CL, Carroll MD, Kit BK et al. (2014) Prevalence of childhood and adult obesity in the United States, 2011-2012. JAMA 311, 806-814.

4. Hales CM, Carroll MD, Fryar CD et al. (2017) Prevalence of Obesity among Adults and Youth: United States, 2015-2016. NCHS data brief, 1-8.

5. Villareal DT, Apovian CM, Kushner RF et al. (2005) Obesity in older adults: technical review and position statement of the American Society for Nutrition and NAASO, the obesity society. Am J Clin Nutr 82, 923-934.

6. Samper-Ternent R \& Al Snih S (2012) Obesity in older adults: epidemiology and implications for disability and disease. Rev Clin Gerontol 22, 10-34.

7. Kimmons JE, Blanck HM, Tohill BC et al. (2006) Associations between body mass index and the prevalence of low micronutrient levels among US adults. MedGenMed 8, 59.

8. Kaidar-Person O, Person B, Szomstein S et al. (2008) Nutritional deficiencies in morbidly obese patients: a new form of malnutrition? Part A: vitamins. Obes Surg 18, 870-876.

9. Kaidar-Person O, Person B, Szomstein S et al. (2008) Nutritional deficiencies in morbidly obese patients: a new form of malnutrition? Part B: minerals. Obes Surg 18, 1028-1034.

10. Agarwal S, Reider C, Brooks JR et al. (2015) Comparison of prevalence of inadequate nutrient intake based on body weight status of adults in the United States: an analysis of NHANES 2001-2008. J Am Coll Nutr 34, 126-134.

11. Inzitari M, Doets E, Bartali B et al. (2011) Nutrition in the age-related disablement process. J Nutr Health Aging 15, 599-604.

12. Sheean P, Farrar IC, Sulo S et al. (2019) Nutrition risk among an ethnically diverse sample of community-dwelling older adults. Public Health Nutr 22, 894-902.

13. Ledikwe JH, Smiciklas-Wright H, Mitchell DC et al. (2003) Nutritional risk assessment and obesity in rural older adults: a sex difference. Am J Clin Nutr 77, 551-558.

14. Eggersdorfer M, Akobundu U, Bailey RL et al. (2018) Hidden hunger: solutions for America's aging populations. Nutrients 10, 1210.

15. Castaneda-Gameros D, Redwood S \& Thompson JL (2017) Low nutrient intake and frailty among overweight and obese migrant women from ethnically diverse backgrounds ages 60 years and older: a mixed-methods study. J Nutr Educ Behav 49, 3-10 e11.

16. Semba RD, Bartali B, Zhou J et al. (2006) Low serum micronutrient concentrations predict frailty among older women living in the community. J Gerontol A Biol Sci Med Sci 61, 594-599.

17. Ames BN (2018) Prolonging healthy aging: longevity vitamins and proteins. Proc Natl Acad Sci 115, 10836-10844.
18. National Center for Health Statistics (2014) National Health and Nutrition Examination Survey: Sample Design, 2011-2014. Washington, DC: Vital Health Stat.

19. National Center for Health Statistics (2018) National Health and Nutrition Examination Survey: Analytic Guidelines, 2011-2014 and 2015-2016. https://wwwn.cdc.gov/nchs/ data/nhanes/analyticguidelines/17-18-sampling-variabilitynonresponse-508.pdf (accessed March 2019).

20. National Institutes of Health (1998) Clinical guidelines on the identification, evaluation, and treatment of overweight and obesity in adults - the evidence report. Obes Res 6, Suppl. 2, 51s-209s.

21. U.S. Department of Health and Human Sciences (2019) HHS Poverty Guidelines and Federal Register References. https://aspe.hhs.gov/prior-hhs-poverty-guidelines-and-federalregister-references (accessed 2019 March 27).

22. Oliveira V (2018) The Food Assistance Landscape: FY 2017 Annual Report. Washington DC: U.S. Department of Agriculture Economic Research Service.

23. Bickel G, Nord M, Price C et al. (2010) Measuring Food Security in the United States: Guide to Measuring Household Food Insecurity. Alexandria, VA: Food and Nutrition Service, U.S. Department of Agriculture.

24. Gandhi K, Lim E, Davis J et al. (2017) Racial-ethnic disparities in self-reported health status among US adults adjusted for sociodemographics and multimorbidities, National Health and Nutrition Examination Survey 2011-2014. Ethn Health 25, 65-78.

25. Kroenke K, Spitzer RL \& Williams JB (2001) The PHQ-9: validity of a brief depression severity measure. $J$ Gen Intern Med 16, 606-613.

26. Kantor ED, Rehm CD, Haas JS et al. (2015) Trends in prescription drug use among adults in the United States from 1999-2012. JAMA 314, 1818-1831.

27. Vasquez E, Batsis JA, Germain CM et al. (2014) Impact of obesity and physical activity on functional outcomes in the elderly: data from NHANES 2005-2010. J Aging Health 26, 1032-1046.

28. World Health Organization (1994) Assessment of fracture risk and its application to screening for postmenopausal osteoporosis: report of a WHO Study Group. https://apps. who.int/iris/handle/10665/39142 (accessed March 2019).

29. Alberti KG, Eckel RH, Grundy SM et al. (2009) Harmonizing the metabolic syndrome: a joint interim statement of the International Diabetes Federation Task Force on Epidemiology and Prevention; National Heart, Lung, and Blood Institute; American Heart Association; World Heart Federation; International Atherosclerosis Society; and International Association for the Study of Obesity. Circulation 120, $1640-1645$.

30. Blanton CA, Moshfegh AJ, Baer DJ et al. (2006) The USDA Automated Multiple-Pass Method accurately estimates group total energy and nutrient intake. J Nutr 136, 2594-2599.

31. U.S. Department of Health and Human Services \& U.S. Department of Agriculture (2015) 2015-2020 Dietary Guidelines for Americans. https://health.gov/dietaryguidelines/ 2015/guidelines/ (accessed March 2019).

32. Russell RM (2001) Factors in aging that effect the bioavailability of nutrients. J Nutr 131, 1359s-1361s.

33. Ervin RB \& Kennedy-Stephenson J (2002) Mineral intakes of elderly adult supplement and non-supplement users in the third national health and nutrition examination survey. J Nutr 132, 3422-3427.

34. Bailey RL, Dodd KW, Gahche JJ et al. (2019) Best practices for dietary supplement assessment and estimation of total usual nutrient intakes in population-level research and monitoring. J Nutr 149, 181-197.

35. Bailey RL, Akabas SR, Paxson EE et al. (2017) Total usual intake of shortfall nutrients varies with poverty among US adults. J Nutr Educ Behav 49, 639-646.e633. 
36. Institute of Medicine (2000) Dietary Reference Intakes: Applications in Dietary Assessment. Washington, DC: National Academies Press (US).

37. Krebs-Smith SM, Pannucci TE, Subar AF et al. (2018) Update of the Healthy Eating Index: HEI-2015. J Acad Nutr Diet 118 , $1591-1602$.

38. Zhang S, Midthune D, Guenther PM et al. (2011) A new multivariate measurement error model with zero-inflated dietary data, and its application to dietary assessment. Ann Appl Stat 5, 1456-1487.

39. Reedy J, Lerman JL, Krebs-Smith SM et al. (2018) Evaluation of the Healthy Eating Index-2015. J Acad Nutr Diet 118 , $1622-1633$.

40. National Cancer Institute (2019) The Healthy Eating Index: SAS Code. https://epi.grants.cancer.gov/hei/sas-code.html (accessed October 2018)

41. Center for Disease Control and Prevention. NHANES 2011-2012 Laboratory Methods. https://wwwn.cdc.gov/nchs/nhanes/ continuousnhanes/labmethods.aspx?BeginYear=2011 (accessed March 2019).

42. Center for Disease Control and Prevention. NHANES 2013-2014 Laboratory Methods. https://wwwn.cdc.gov/nchs/nhanes/ continuousnhanes/labmethods.aspx?BeginYear=2013 (accessed March 2019).

43. Institute of Medicine Standing Committee on the Scientific Evaluation of Dietary Reference Intakes (1998) Dietary Reference Intakes for Thiamin, Riboflavin, Niacin, Vitamin B6, Folate, Vitamin B12, Pantothenic Acid, Biotin, and Choline. Washington, DC: National Academies Press.

44. Allen LH, Miller JW, de Groot L et al. (2018) Biomarkers of Nutrition for Development (BOND): vitamin B-12 review. J Nutr 148, 1995S-2027S

45. Vogiatzoglou A, Oulhaj A, Smith AD et al. (2009) Determinants of plasma methylmalonic acid in a large population: implications for assessment of vitamin B12 status. Clin Chem 55, 2198-2206.

46. Levey AS, Stevens LA, Schmid CH et al. (2009) A new equation to estimate glomerular filtration rate. Ann Intern Med 150, 604-612.

47. Flamant M, Haymann JP, Vidal-Petiot E et al. (2012) GFR estimation using the Cockcroft-Gault, MDRD study, and CKD-EPI equations in the elderly. AJKD 60, 847-849.

48. Levey AS, Coresh J, Balk E et al. (2003) National Kidney Foundation practice guidelines for chronic kidney disease: evaluation, classification, and stratification. Ann Intern Med 139, 137-147.

49. Centers for Disease Control and Prevention Chronic Kidney Disease Surveillance System - United States. http://www. cdc.gov/ckd (accessed June 2019).

50. Institute of Medicine (2011) Dietary Reference Intakes for Calcium and Vitamin D. Washington, DC: National Academies Press (US).

51. Dawson-Hughes B (2004) Racial/ethnic considerations in making recommendations for vitamin $\mathrm{D}$ for adult and elderly men and women. Am J Clin Nutr 80, 1763s-1766s.

52. Gahche JJ, Bailey RL, Potischman N et al. (2017) Dietary supplement use was very high among older adults in the United States in 2011-2014. J Nutr 147, 1968-1976.

53. Bailey RL, Fulgoni VL, 3rd, Keast DR et al. (2011) Dietary supplement use is associated with higher intakes of minerals from food sources. Am J Clin Nutr 94, 1376-1381.

54. Bailey RL, Fulgoni VL, 3rd, Keast DR et al. (2012) Examination of vitamin intakes among US adults by dietary supplement use. J Acad Nutr Diet 112, 657-663 e654.

55. Blumberg JB, Frei B, Fulgoni VL et al. (2017) Contribution of dietary supplements to nutritional adequacy in various adult age groups. Nutrients $\mathbf{9}, 1325$.

56. Thomas-Valdes S, Tostes M, Anunciacao PC et al. (2017) Association between vitamin deficiency and metabolic disorders related to obesity. Crit Rev Food Sci Nutr 57, 3332-3343.
57. Nielsen FH (2010) Magnesium, inflammation, and obesity in chronic disease. Nutr Rev 68, 333-340.

58. Volpe SL (2013) Magnesium in disease prevention and overall health. Adv Nutr $\mathbf{4}, 378$ s-383s.

59. Institute of Medicine (1997) Dietary Reference Intakes for Calcium, Phosphorus, Magnesium, Vitamin D, and Fluoride. Washington, DC: National Academies Press.

60. Subar AF, Freedman LS, Tooze JA et al. (2015) Addressing current criticism regarding the value of self-report dietary data. J Nutr 145, 2639-2645.

61. Lissner L, Troiano RP, Midthune D et al. (2007) OPEN about obesity: recovery biomarkers, dietary reporting errors and BMI. Int J Obes (Lond) 31, 956-961.

62. Lagunova Z, Porojnicu AC, Lindberg F et al. (2009) The dependency of vitamin D status on body mass index, gender, age and season. Anticancer Res 29, 3713-3720.

63. Parikh SJ, Edelman M, Uwaifo GI et al. (2004) The relationship between obesity and serum 1,25-dihydroxy vitamin D concentrations in healthy adults. J Clin Endocrinol Metab 89, 1196-1199.

64. Snijder MB, van Dam RM, Visser M et al. (2005) Adiposity in relation to vitamin D status and parathyroid hormone levels: a population-based study in older men and women. J Clin Endocrinol Metab 90, 4119-4123.

65. Walsh JS, Evans AL, Bowles S et al. (2016) Free 25-hydroxyvitamin $\mathrm{D}$ is low in obesity, but there are no adverse associations with bone health. Am J Clin Nutr 103, 1465-1471.

66. Herrick KA, Storandt RJ, Afful J et al. (2019) Vitamin D status in the United States, 2011-2014. Am J Clin Nutr 110, 150-157.

67. Vanlint S (2013) Vitamin D and obesity. Nutrients 5, 949-956.

68. Drincic AT, Armas LA, Van Diest EE et al. (2012) Volumetric dilution, rather than sequestration best explains the low vitamin D status of obesity. Obesity (Silver Spring) 20, 1444-1448.

69. Bailey RL, Fulgoni VL, Taylor CL et al. (2017) Correspondence of folate dietary intake and biomarker data. Am J Clin Nutr 105, 1336-1343.

70. Bird JK, Ronnenberg AG, Choi SW et al. (2015) Obesity is associated with increased red blood cell folate despite lower dietary intakes and serum concentrations. J Nutr 145, 79-86.

71. Mojtabai R (2004) Body mass index and serum folate in childbearing age women. Eur J Epidemiol 19, 1029-1036.

72. Mahabir S, Ettinger S, Johnson L et al. (2008) Measures of adiposity and body fat distribution in relation to serum folate levels in postmenopausal women in a feeding study. Eur J Clin Nutr 62, 644-650.

73. Mineva EM, Sternberg MR, Zhang M et al. (2019) Age-specific reference ranges are needed to interpret serum methylmalonic acid concentrations in the US population. Am J Clin Nutr 110, 158-168.

74. Sun Y, Sun M, Liu B et al. (2019) Inverse association between serum vitamin $\mathrm{B}_{12}$ concentration and obesity among adults in the United States. Front Endocrinol 10, 414.

75. Allin KH, Friedrich N, Pietzner M et al. (2017) Genetic determinants of serum vitamin B12 and their relation to body mass index. Eur J Epidemiol 32, 125-134.

76. Shlisky J, Bloom DE, Beaudreault AR et al. (2017) Nutritional considerations for healthy aging and reduction in age-related chronic disease. Adv Nutr 8, 17-26.

77. Garcia OP, Long KZ \& Rosado JL (2009) Impact of micronutrient deficiencies on obesity. Nutr Rev 67, 559-572.

78. Moshfegh AJ, Rhodes DG, Baer DJ et al. (2008) The US Department of Agriculture Automated Multiple-Pass Method reduces bias in the collection of energy intakes. Am J Clin Nutr 88, 324-332.

79. Raghavan R, Ashour FS \& Bailey R (2016) A review of cutoffs for nutritional biomarkers. Adv Nutr 7, 112-120.

80. Zamboni M, Mazzali G, Zoico E et al. (2005) Health consequences of obesity in the elderly: a review of four unresolved questions. Int J Obes (Lond) 29, 1011-1029. 